\title{
On the X-ray fast-time variability of Sco X-2 (GX 349+2)
}

\author{
P. M. O’Neill ${ }^{1}$, E. Kuulkers ${ }^{2,3}$, R. K. Sood ${ }^{1}$, and T. Dotani ${ }^{4}$ \\ 1 School of Physics, Australian Defence Force Academy, University of New South Wales, \\ Canberra ACT 2600, Australia \\ e-mail: r.sood@adfa.edu .au \\ 2 Space Research Organization Netherlands, Sorbonnelaan 2, 3584 CA Utrecht, The Netherlands \\ e-mail: erikk@sron.nl \\ 3 Astronomical Institute, Utrecht University, PO Box 80000, 3507 TA Utrecht, The Netherlands \\ 4 Institute of Space and Astronautical Science, 1-1 Yoshinodai 3-chome, Sagamihara-shi, \\ Kanagawa 229-8510, Japan \\ e-mail: dotani@astro.isas.ac.jp
}

Received 11 October 2000 / Accepted 2 February 2001

\begin{abstract}
We have analysed archived Ginga data on the Z source Sco X-2 (GX 349+2). We present the first detailed investigation of its X-ray fast-time variability, as a function of position in the Z track. During the two-day observation over the period 5-7 March 1989, the source was in the so-called flaring branch, and the lower part of the so-called normal branch. We found broad peaked noise with a centroid frequency and width of $\sim 4-7 \mathrm{~Hz}$ and $\sim 6-12 \mathrm{~Hz}$ respectively. The peaked noise was strongest in the lower flaring branch, with a maximum fractional rms amplitude of $\sim 3 \%$. We conclude that it is not a manifestation of atoll source high frequency noise, as had been suggested, and compare it with the power spectral features seen in other $\mathrm{Z}$ sources. We find that the peaked noise is markedly different to the quasi-periodic oscillations found in the normal and flaring branches of Sco X-1; however it bears some resemblance to that seen in the flaring branch of Cyg X-2 at low overall intensities.
\end{abstract}

Key words. accretion, accretion disks - stars: binaries: close - stars: individual: Sco X-2; GX 349+2 stars: neutron - X-rays: stars

\section{Introduction}

Sco X-2 (GX $349+2)$ is a neutron star low-mass X-ray binary and $\mathrm{Z}$ source. $\mathrm{Z}$ sources trace out a characteristic "Z" shaped track in an X-ray colour-colour diagram (CD) or hardness-intensity diagram (HID) consisting of a "horizontal" branch (HB), "normal" branch (NB) and "flaring" branch (FB) (Hasinger \& van der Klis 1989; hereafter HK; van der Klis 1995). The orientation of each branch in a CD or HID depends upon the choice of energy bands. Mass-accretion rate $(\dot{M})$ is thought to increase from the $\mathrm{HB}$, down the NB and up the FB. The other traditional Z sources are: Sco X-1, GX 17+2, GX 340+0, Cyg X-2, and GX 5-1.

A class of objects that are related to $\mathrm{Z}$ sources are the "atoll" sources. Atoll sources trace out a curved track in a $\mathrm{CD}$, with inferred mass-accretion rate increasing along the track. Atoll sources are thought to have lower magnetic field strengths, and lower mass-accretion rates than $\mathrm{Z}$ sources (HK).

Send offprint requests to: P. M. O'Neill, e-mail: p.m.oneill@adfa.edu.au
The X-ray fast timing properties of $\mathrm{Z}$ and atoll sources are different, yet related. The features observed in the power spectra of both classes are normally correlated with the position of the source in a CD or HID. Z sources exhibit very low frequency noise (VLFN), low frequency noise (LFN), high frequency noise (HFN), and quasiperiodic oscillations (QPOs). QPOs with frequencies in the range $15-60 \mathrm{~Hz}$ (called HBOs) and $200-1200 \mathrm{~Hz}(\mathrm{kHz}$ QPOs; these typically occur in pairs) are present in the HB and upper part of the NB. Atoll sources exhibit VLFN, HFN, 1-70 Hz QPOs and kHz QPOs. Atoll source HFN can sometimes be peaked, and resemble broad QPOs (HK and references therein). A recent review of the fast-time variability of $\mathrm{Z}$ and atoll sources, with a discussion of various models, has been given by van der Klis (2000).

In Z sources, narrow QPOs (called NBOs) with centroid frequencies in the range $5-8 \mathrm{~Hz}$ have been observed in the NB. NBOs typically become detectable halfway down the NB, and their properties differ between objects. In Sco X-1 the frequency of the NBO is $\sim 5.5-8 \mathrm{~Hz}$ in the $\mathrm{NB}$, and jumps to $\sim 12 \mathrm{~Hz}$ as the source enters the $\mathrm{FB}$ (the $\mathrm{QPO}$ is then referred to as an $\mathrm{FBO}$ ) 
(Dieters \& van der Klis 2000). As the source moves up the $\mathrm{FB}$, the frequency and full width at half maximum $(F W H M)$ increase; eventually, at $\sim 10 \%$ of the way up the $\mathrm{FB}$, the FBO becomes so broad as to be indistinguishable from the underlying noise (but the relative width never exceeds $\sim 0.5)$. In Sco X-1 the fractional rms increases with increasing $\dot{M}$ and is greater at higher energies; and there is no time lag between low and high energy bands (Dieters et al. 2000). The $\mathrm{N} / \mathrm{FBO}$ seen in GX $17+2$ behaves in a similar fashion to Sco X-1 (Penninx et al. 1990). In the other Z sources NBOs have also been observed but are generally less prominent (e.g. Kuulkers et al. 1994).

Sco X-2 is seemingly very similar to Sco X-1. The $\mathrm{Z}$ tracks have nearly identical shapes (except for the absence of an $\mathrm{HB}$ in Sco X-2) and the lightcurves of both objects exhibit strong flaring behaviour (Schultz et al. 1989; Kuulkers \& van der Klis 1995a). The orbital periods are also similar: $18.9 \mathrm{~h}$ for Sco $\mathrm{X}-1$ (Cowley \& Crampton 1975); and $\sim 22 \mathrm{~h}$ for Sco $\mathrm{X}-2$ (Wachter 1997). One might therefore expect the $\mathrm{X}$-ray fast-time variability in Sco X-2 to resemble that seen in Sco X-1; but this is not the case.

EXOSAT observations of Sco X-2 reported by Ponman et al. (1988) showed a complete FB. No narrow NBO was found; however broad peaked noise was detected with a centroid frequency and $F W H M$ of about $5 \mathrm{~Hz}$ and $10 \mathrm{~Hz}$ respectively. The peaked noise was strongest in the lower FB, with a fractional rms of $3.2 \pm 0.2 \%$. There was no significant change in the frequency or width of the peaked noise, and there was no time lag between the low and high energy bands. We note that the authors divided their data into four segments on the basis of intensity, and not according to position in the $\mathrm{Z}$ track. By using that method they could not differentiate between the NB and the lower FB.

A small set of Rossi X-ray Timing Explorer observations were reported by Kuulkers \& van der Klis (1998; hereafter KK), when Sco X-2 was near the NB/FB vertex. In the lower NB they found peaked noise with a centroid frequency, $F W H M$ and fractional rms of $9.4 \pm$ $0.5 \mathrm{~Hz}, 16 \pm 2 \mathrm{~Hz}$ and $2.7 \pm 0.1 \%$ respectively. In the lower region of the $\mathrm{FB}$ they measured these values as $5.8 \pm 0.2 \mathrm{~Hz}, 11.0 \pm 0.7 \mathrm{~Hz}$ and $4.2 \pm 0.1 \%$. There was a small but significant decrease in frequency when the source moved through the NB/FB vertex. They commented that the peaked noise resembled the peaked HFN sometimes seen in atoll sources. They found that the peaked noise fractional rms was stronger at higher energies.

Even though there have been clear indications that Sco X-2 exhibits marked differences to Sco X-1, it has remained largely ignored. This is perhaps due to the fact that it looks so similar to Sco X-1 in a CD and HID.

We present results obtained from an analysis of archived data from the Ginga satellite; these data have not previously been presented. This work is the first detailed analysis of the power spectral properties of Sco X-2 as a function of position in the $\mathrm{Z}$ track. This analysis method is extremely useful because it allows us to unambiguously distinguish between the NB and lower FB, and is helpful when making comparisons between different objects. We further highlight the differences between Sco X-2 and Sco X-1, and conclude that the observed peaked noise is not the same as atoll source peaked HFN, as had been suggested. We compare our results with the power spectral features seen in other Z sources.

\section{Observations}

The Ginga Large Area Counter (LAC) (Makino \& ASTRO-C Team 1987; Turner et al. 1989) observed Sco X-2 between 5 and 7 March 1989. We retrieved the data from the Leicester Database and Archive Service ${ }^{1}$. In Table 1 we present a log of observations.

The LAC consisted of eight detectors, and was sensitive to $\mathrm{X}$-rays in the range $1-37 \mathrm{keV}$. Several modes were available, with differing time and energy resolutions (higher time resolution sacrifices energy resolution). The observations discussed here were done in the so-called MPC3 and PC modes. For the MPC3 mode the coarse gain setting was high, providing an energy range 1-19 keV over twelve channels. The time resolution was $7.8 \mathrm{~ms}$ and $62.5 \mathrm{~ms}$ for high (MPC3-H) and medium (MPC3-M) bit rates respectively. In the $\mathrm{PC}$ mode, the $\mathrm{LAC}$ was divided into two groups of four detectors each. The coarse gain setting was low, giving an energy range 1.5-24.3 keV for one detector group, and 1.2-17.9 keV for the other. Each detector group had two energy channels, so the PC mode observations had four overlapping energy bands. In the high bit rate mode $(\mathrm{PC}-\mathrm{H})$, the time resolutions were $0.98 \mathrm{~ms}$ and $1.95 \mathrm{~ms}$ for the lower and higher energy bands respectively. Our $0.98 \mathrm{~ms}$ data were rebinned with a time resolution of $1.95 \mathrm{~ms}$, to obtain lightcurves for the full energy range.

During the observations in Sect. C (see Table 1) the high voltages in detectors 5 and 6 were accidently changed. The energy range for the MPC3 observations stated above is therefore only approximate for Sect. C.

\section{Analysis}

The counting rates were corrected for detector deadtime and collimator response. During the observations in Sects. A-C, the collimator response was $\sim 0.96$. In Sect. D the response was $\sim 0.73$. The data were not corrected for collimator reflection. The effect of collimator reflection was to overestimate counting rates below $\sim 6 \mathrm{keV}$ by a few percent (Turner et al. 1989; see also Hasinger et al. 1990; Wijnands et al. 1997).

Hardness-intensity diagrams were constructed using $64 \mathrm{~s}$ averages. For the MPC3 data we defined the hardness as the counting rate ratio between $8.2-16.7 \mathrm{keV}$ and $5.8-8.2 \mathrm{keV}$. The intensity was defined as the counting rate in the range $1.1-16.7 \mathrm{keV}$. For the $\mathrm{PC}$ data we defined the hardness as the counting rate ratio between $5.7-17.9 \mathrm{keV}$

\footnotetext{
1 see http://ledas-www.star.le.ac.uk/ginga/
} 
Table 1. Observation $\log$

\begin{tabular}{cccccc}
\hline Section & Start Time (UT) & End Time (UT) & Usable Data (s) & Modes & Time Res (ms) \\
\hline A & 5 Mar. 1989 20:58 & 6 Mar. 1989 00:17 & 4512 & PC-H & $0.98 / 1.95$ \\
B & 6 Mar. 1989 00:27 & 6 Mar. 1989 19:13 & 2880 & MPC3-H & 7.8 \\
& & & 12928 & MPC3-M & 62.5 \\
C & 7 Mar. 1989 02:13 & 7 Mar. 1989 14:30 & 12928 & MPC3-M & 62.5 \\
D & 7 Mar. 1989 18:12 & 7 Mar. 1989 19:14 & 1184 & PC-H & $0.98 / 1.95$ \\
\hline
\end{tabular}

and $1.2-5.7 \mathrm{keV}$. The intensity was defined as the counting rate, from one detector group only (i.e. four detectors), in the range $1.2-17.9 \mathrm{keV}$.

Timing analysis was performed on the raw (i.e. not corrected for deadtime and collimator response) data. Power spectra were calculated from $16 \mathrm{~s}$ intervals in several energy bands, and then averaged according to either corrected counting rate or rank number.

Rank number was introduced by Hasinger et al. (1990) (see also Lewin et al. 1992; Hertz et al. 1992; Dieters \& van der Klis 2000) and is a one-dimensional measure of the position of the source in a CD or HID. Rank number increases smoothly with increasing inferred mass-accretion rate. To measure the rank numbers, a spline was fitted through the $\mathrm{Z}$ track, and each point was projected onto it. Rank number was then determined by measuring the distance of each point along the spline. We defined the $\mathrm{NB} / \mathrm{FB}$ vertex as rank number 1 , and the top of the observed FB as rank number 2.

We calculated the expected deadtime-affected whitenoise levels and then subtracted the white-noise from each average power spectrum. The procedure to calculate white-noise levels in sum channel power spectra is well known, and is described by van der Klis (1989) and Mitsuda \& Dotani (1989). The full procedure to calculate white-noise levels in energy resolved power spectra is not explicity stated in those references; we therefore describe our procedure in the Appendix.

After the deadtime-affected white-noise levels had been subtracted, the spectra were normalised to fractional-rms-squared per $\mathrm{Hz}$ and functional $\chi^{2}$ fits were performed. Very low frequency noise (VLFN) was modelled using a power law $A \nu^{\alpha}$ and integrated over the range 0.01-1 Hz. Peaked noise was modelled using a Lorentzian. All power spectra were initially fitted with only a power law. The significance of adding a Lorentzian component to the fit was then estimated via an F-test. If the inclusion of a peaked noise component was not significant $(<3 \sigma)$ we measured $90 \%$ confidence upper limits by using $\Delta \chi^{2}=2.71$. Errors on all parameters were measured using $\Delta \chi^{2}=1$. When a fit was not sensitive to the centroid or $F W H M$ of the Lorentzian (or was not significantly improved by its inclusion) we fixed those parameters at values measured from other power spectra (the actual values are shown in Table 2). We fitted each PC mode power spectrum both with and without an extra free constant component. The reasons for doing this are discussed in Sect. 4.3 .

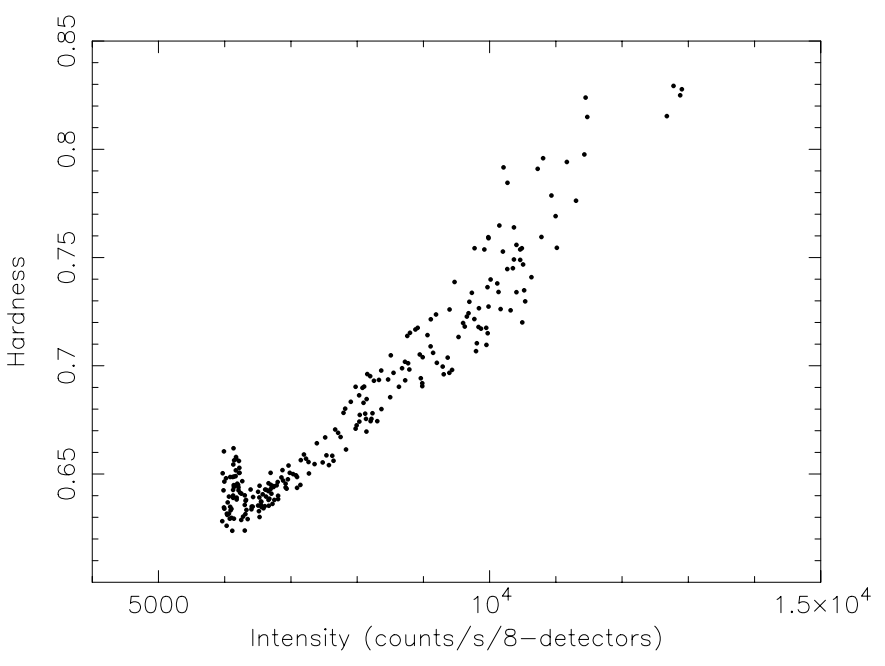

Fig. 1. HID of Sect. B, with $64 \mathrm{~s}$ averages. The intensity is the counting rate in the range $1.1-16.7 \mathrm{keV}$. The hardness is the counting rate ratio between $8.2-16.7 \mathrm{keV}$ and $5.8-8.2 \mathrm{keV}$. The error bars are slightly larger than the dots

All fractional rms amplitudes were corrected for channel cross-talk (Lewin et al. 1992). We assumed variations at all energies were in phase. Finally, the fractional rms values were multiplied by a binning correction factor (van der Klis 1989).

\section{Results}

\subsection{Hardness-intensity diagrams}

Figure 1 shows a HID for all Sect. B data. The small, approximately vertical NB is clearly visible. Sco X-2 is known to flare to intensities roughly twice the level of the persistent emission. The highest intensity in Fig. 1 is a factor of 2.2 greater than the lowest intensity: we conclude therefore, that we have observed a fully developed flaring branch. The results from Sect. C are compatible with those from Sect. B; however due to the uncertain energy ranges of those data, we will not discuss them any further.

A HID of sections A and D is shown in Fig. 2. The lowest and highest intensities in Fig. 2 differ by a factor of 2 ; therefore (in comparison with Fig. 1), nearly all of the FB was traced out. No clear NB/FB vertex is apparent (but we were able to estimate where it might lie; see Sect. 4.3). When we constructed a HID from MPC3 mode data using energy bands similar to those available in PC mode, the presence of the NB was much less pronounced; the NB became more like a "blob", rather than 


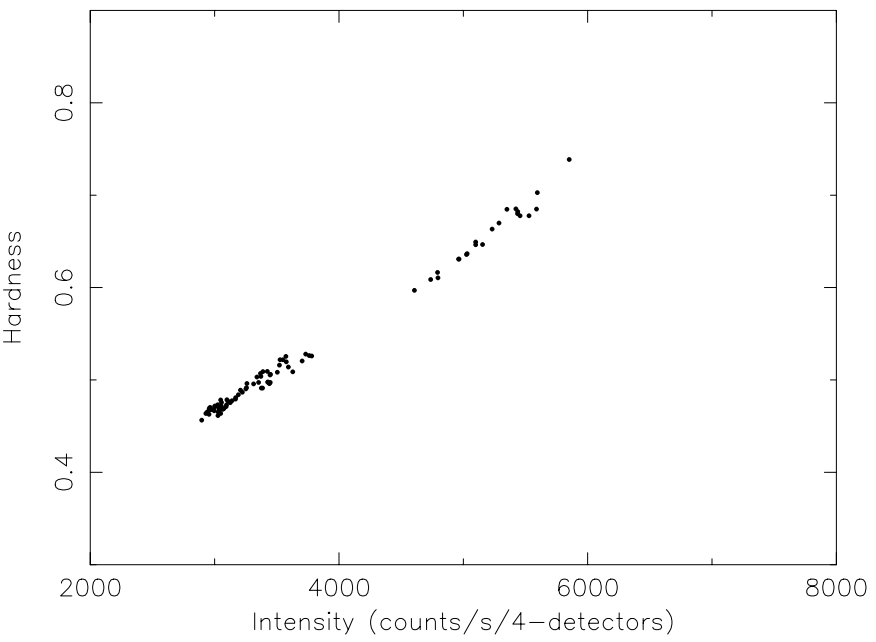

Fig. 2. HID of Sects. A and D, with $64 \mathrm{~s}$ averages. The intensity is the counting rate in the range $1.2-17.9 \mathrm{keV}$. The hardness is the counting rate ratio between $5.7-17.9 \mathrm{keV}$ and $1.2-5.7 \mathrm{keV}$. The error bars are slightly larger than the dots

a clear branch. Therefore we caution that in Fig. 2 we cannot confidently distinguish between data points from the lower NB (if there are any) and those actually in the lower FB. However, as will be seen in Sect. 4.3, our main interest (with the PC data) is with the top half of the FB, so this ambiguity is not a major problem.

\subsection{Timing analysis of section B MPC3 mode data}

During the observations in MPC3-H mode, the source was found only in the lower FB. We divided the data into two segments with mean ranks $1.09 \pm 0.02$ and $1.26 \pm 0.05$. An example of a power spectrum $(1.1-16.7 \mathrm{keV})$ is shown in Fig. 3, with mean rank number 1.09. The results of fitting the MPC3-H data $(1.1-16.7 \mathrm{keV})$ are shown in Table 2. The $F W H M$ of the best-fit Lorentzian is greater than half its centroid frequency, so the feature cannot be formally described as a QPO. The MPC3-H data show that there is no significant change in the centroid frequency or $F W H M$ of the peaked noise, as the source moves up the FB (the decrease in the centroid frequency between rank numbers 1.09 and 1.26 is significant only at the $\sim 2 \sigma$ level). The fractional rms amplitude of the peaked noise is also consistent with remaining constant.

We investigated the energy dependence of the peaked noise fractional rms, by calculating energy resolved power spectra from the MPC3-H data. We used four energy bands: $1.1-3.4-5.8-8.2-16.7 \mathrm{keV}$. All power spectra from a particular band were averaged together, giving a mean power spectrum from the lower FB; the mean rank number was $1.18 \pm 0.05$. We determined the centroid frequency and $F W H M$ of the peaked noise feature from an average power spectrum from the $1.1-16.7 \mathrm{keV}$ band: they were $6.7 \pm 0.4 \mathrm{~Hz}$ and $8.3_{-1.1}^{+1.2} \mathrm{~Hz}$ respectively. The centroid and width were fixed at those values when fitting the power spectra from the other four energy bands. The energy dependence of peaked noise fractional rms is shown in

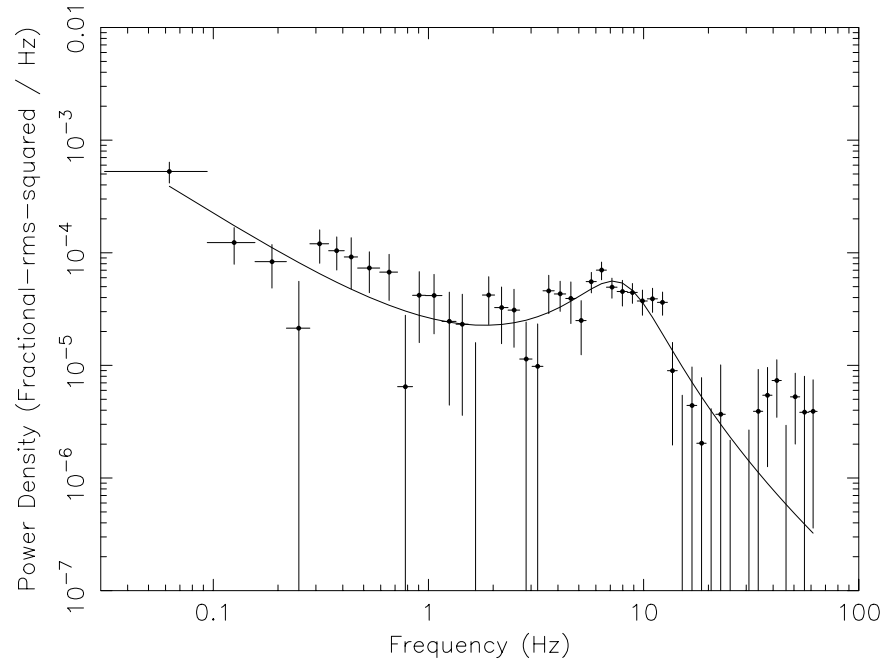

Fig. 3. MPC3-H power spectrum from the energy range 1.1-16.7 keV. The mean rank is 1.09 (lower FB). The solid line shows the best fit

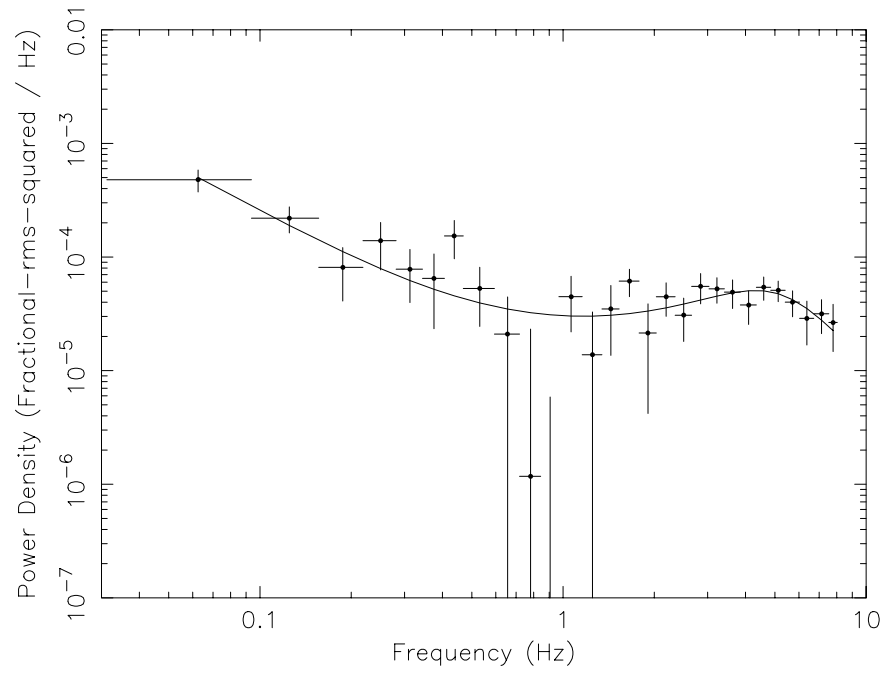

Fig. 4. MPC3-M power spectrum from the energy range 1.1-16.7 keV. The mean rank is 1.09 (lower FB). The solid line shows the best fit

Table 3 . The peaked noise is clearly stronger at higher energies and is consistent with being constant above $\sim 6 \mathrm{keV}$.

The MPC3-M data from the track shown in Fig. 1 were divided into eight segments. Power spectra were averaged on the basis of rank number. An example (1.1-16.7 keV) is shown in Fig. 4, with mean rank number 1.09. Table 2 shows the results of fitting the MPC3-M power spectra $(1.1-16.7 \mathrm{keV})$ from Sect. B. It was only in the lower FB that we could meaningfully constrain the centroid and width of the peaked noise, which were $\sim 4 \mathrm{~Hz}$ and $\sim 6 \mathrm{~Hz}$ respectively. There is no significant change in those values between mean ranks 1.09 and 1.27 (Table 2). The centroid frequency is lower in the MPC3-M data than in the MPC3-H data. This discrepancy is probably due to a lack of information above $8 \mathrm{~Hz}$ in the MPC3-M data.

The dependence of peaked noise and VLFN fractional rms on rank number is shown in Figs. 5 and 6 respectively. 
Table 2. Results from fits to the Sect. B MPC3 power spectra from the energy range 1.1-16.7 keV

\begin{tabular}{ccccccc}
\hline Mean Rank & \multicolumn{2}{c}{ VLFN } & \multicolumn{3}{c}{ Peaked Noise } & Reduced $\chi^{2} /$ dof \\
& Index & rms (\%) & Frequency $(\mathrm{Hz})$ & $F W H M(\mathrm{~Hz})$ & $\mathrm{rms}(\%)$ & \\
\hline MPC3-H data & & & & & & \\
$1.09 \pm 0.02$ & $-1.2_{-0.3}^{+0.2}$ & $1.2_{-0.1}^{+0.2}$ & $7.3 \pm 0.4$ & $7.1_{-1.0}^{+1.2}$ & $2.9 \pm 0.2$ & $1.25 / 43$ \\
$1.26 \pm 0.05$ & $-2.0_{-0.3}^{+0.2}$ & $2.6_{-0.4}^{+0.6}$ & $5.5_{-1.0}^{+0.8}$ & $10.3_{-2.2}^{+3.1}$ & $3.0 \pm 0.3$ & $0.79 / 43$ \\
MPC3-M data & & & & & & \\
$0.95 \pm 0.02$ & $-1.5 \pm 0.3$ & $1.2 \pm 0.2$ & $4.3^{\mathrm{a}}$ & $6.1^{\mathrm{a}}$ & $1.8 \pm 0.2$ & $0.8 / 25$ \\
$1.00 \pm 0.02$ & $-1.2 \pm 0.2$ & $1.3 \pm 0.1$ & $4.3^{\mathrm{a}}$ & $6.1^{\mathrm{a}}$ & $2.3 \pm 0.1$ & $1.28 / 25$ \\
$1.09 \pm 0.03$ & $-1.5 \pm 0.3$ & $1.4 \pm 0.2$ & $4.3 \pm 0.4$ & $6.1_{-1.3}^{+2.1}$ & $2.8 \pm 0.3$ & $0.94 / 23$ \\
$1.27 \pm 0.06$ & $-1.9 \pm 0.2$ & $2.8 \pm 0.5$ & $4.4 \pm 0.6$ & $6.2_{-1.9}^{+2.9}$ & $2.3 \pm 0.3$ & $1.40 / 23$ \\
$1.41 \pm 0.03$ & $-1.6 \pm 0.1$ & $2.9 \pm 0.2$ & $4.4^{\mathrm{a}}$ & $6.2^{\mathrm{a}}$ & $1.4 \pm 0.2$ & $0.84 / 25$ \\
$1.50 \pm 0.03$ & $-1.5 \pm 0.1$ & $2.8 \pm 0.2$ & $4.4^{\mathrm{a}}$ & $6.2^{\mathrm{a}}$ & $<1.3$ & $1.06 / 26$ \\
$1.60 \pm 0.03$ & $-1.6 \pm 0.1$ & $3.3 \pm 0.2$ & $4.4^{\mathrm{a}}$ & $6.2^{\mathrm{a}}$ & $<0.9$ & $1.10 / 26$ \\
$1.75 \pm 0.09$ & $-1.8 \pm 0.1$ & $4.8 \pm 0.3$ & $4.4^{\mathrm{a}}$ & $6.2^{\mathrm{a}}$ & $<1.2$ & $1.10 / 26$ \\
\hline
\end{tabular}

${ }^{\mathrm{a}}$ Fixed parameter.

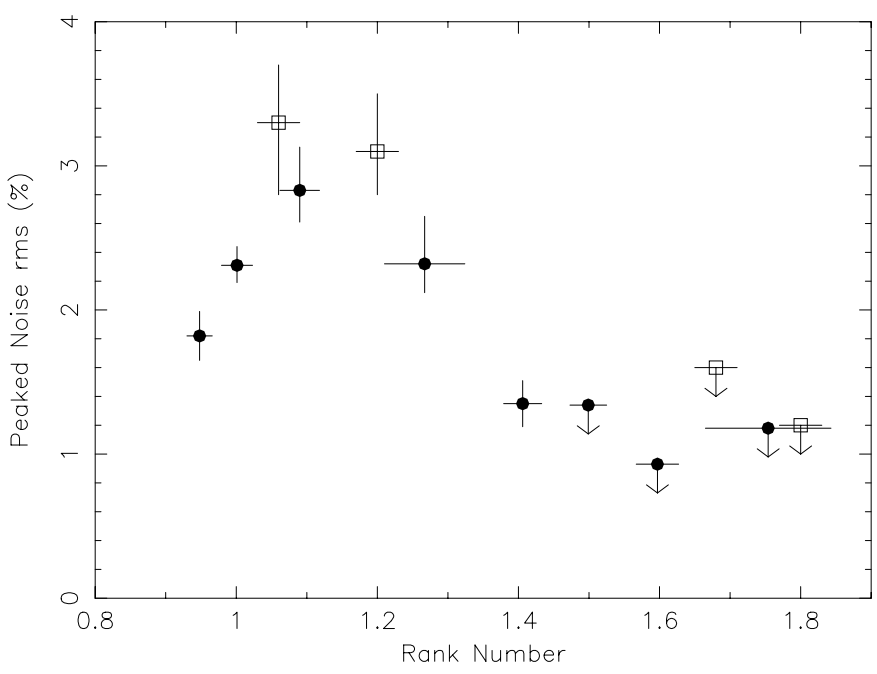

Fig. 5. Peaked noise fractional rms values as a function of rank number for the MPC3-M data (solid dots, 1.1-16.7 keV), and PC data (open squares, $1.2-17.9 \mathrm{keV}$ ). The fractional rms values of the PC data are from fits which included a constant component

The peaked noise fractional rms is greatest in the lower FB and becomes undetectable (upper limit 1.3\%) by about halfway up the FB. Of note is the fact that it is actually detectable up to a mean rank of 1.41. (This is important because N/FBO typically become undetectable by $\sim 10 \%$ of the way up the FB.) The drop in power as the source moves further up the FB or into the NB may be due to the feature moving above the Nyquist frequency of these data; however we can exclude this effect with respect to movement up the FB (see Sect. 4.3). The VLFN fractional $\mathrm{rms}$ is seen to increase as the source moves up the FB, as expected for $\mathrm{Z}$ sources (HK). The slope of the power law appears to be uncorrelated with rank number.
Table 3. Energy dependence of peaked noise fractional rms amplitude

\begin{tabular}{cc}
\hline Energy Range (keV) & rms (\%) \\
\hline $1.1-3.4$ & $<2.8$ \\
$3.4-5.8$ & $<2.6$ \\
$5.8-8.2$ & $4.2_{-0.4}^{+0.3}$ \\
$8.2-16.7$ & $4.8_{-0.5}^{+0.4}$ \\
\hline
\end{tabular}

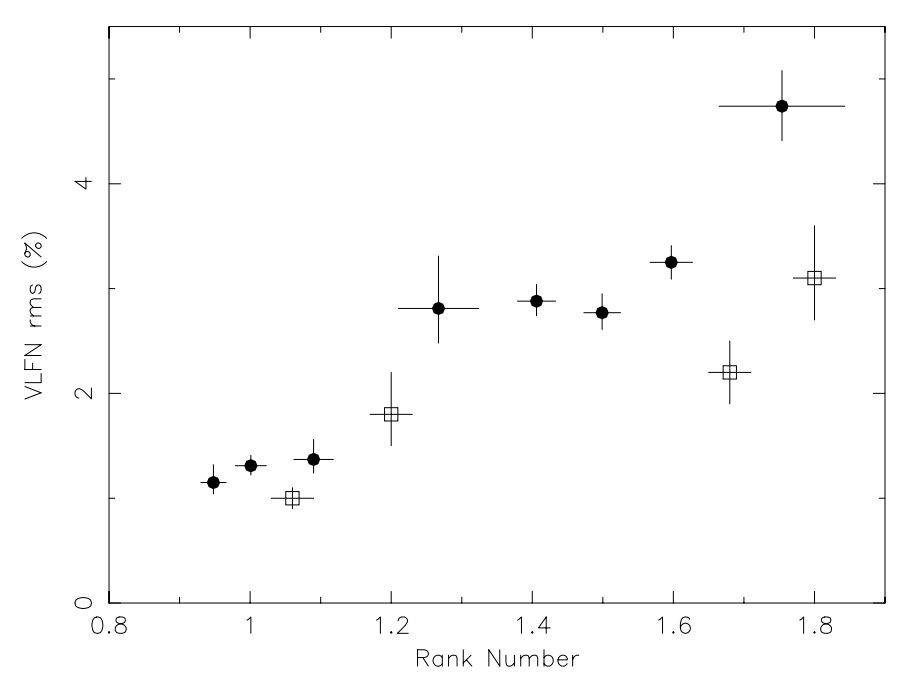

Fig. 6. VLFN fractional rms values as a function of rank number for the MPC3-M data (solid dots, 1.1-16.7 keV), and PC data (open squares, $1.2-17.9 \mathrm{keV}$ ). The fractional rms values of the PC data are from fits which included a constant component

\subsection{Timing analysis of $P C$ mode data}

The NB/FB vertex was not unambiguously observed, so we did not calculate rank numbers for each individual interval; averaging was done on the basis of intensity. The track was divided into four segments; the intensity ranges were: 2800-3200, 3200-3900, 4500-5200 and 52005900 count/s (four detectors only). The mean intensities of those four segments were: $3041 \pm 6,3458 \pm 14,4962 \pm 25$ and $5492 \pm 31$ counts/s. We could, however, estimate rank 
numbers by comparing Fig. 2 with Fig. 1 (assuming the intensities are derived from the same energy band). As stated in Sect. 4.1, the track shown in Fig. 2 is not quite a complete FB. The lowest intensity observed in Fig. 2 is $\sim 2900$ counts/s; this can be taken as the upper limit of the intensity of the NB/FB vertex. Alternatively, if we assume the highest intensity present in Fig. 2 is actually the top of the $\mathrm{FB}$, then the unseen $\mathrm{NB} / \mathrm{FB}$ vertex can be placed at $\sim 2780$ counts $/ \mathrm{s}$; this can be considered to be the lower limit. By considering these two extremes we calculated the approximate rank numbers of the four segments as: $1.06,1.20,1.68$, and $1.80( \pm 0.03)$. We stress that these rank numbers are only a rough guide, and that the lower FB in Fig. 2 may in fact contain data from the NB.

An example power spectrum is shown in Fig. 7 $(1.2-17.9 \mathrm{keV})$, with mean intensity 3041 counts/s (mean rank number $\sim 1.06)$. The results from fitting the $\mathrm{PC}$ power spectra $(1.2-17.9 \mathrm{keV})$ are shown in Table 4 , where we present the fits from models both with and without a constant. The results from fits with a constant are shown also in Figs. 5 and 6 . The most important thing to note is the nondetection of peaked noise in the upper FB (upper limit 1.6\%). The PC data Nyquist frequency is $256 \mathrm{~Hz}$; therefore the nondetection of peaked noise in these data is not due only to the feature moving to higher frequencies. From this, we conclude that the nondetection of peaked noise in the MPC3-M data (Table 2) is also a real weakening of the peak.

The reduced $\chi^{2} \mathrm{~s}$ of the fits without a constant in Table 4 are formally acceptable; however visual inspection of the power spectra revealed excess power above about $100 \mathrm{~Hz}$ (e.g. see Fig. 7), and the best-fit VLFN indices found in the two lowest intensity segments are unusually flat. Given these indications, we attempted to account for a possible high frequency feature. We added a cut-off power law to our model and attempted to fit the spectrum from the lowest intensity segment. We could not constrain the index or cut-off frequency of this component. When we fitted a Lorentzian (instead of the cut-off power law) to the same spectrum we could constrain neither the width nor centroid frequency. The fits were improved with the addition of the extra component, but were not sensitive to the exact form of that component. We fitted the spectrum with a constant (instead of a cut-off power law or Lorentzian) and the fit also improved. None of the best-fit values of the peaked noise or VLFN were sensitive to the type of model that we used to describe the excess power; for simplicity, we therefore chose to use a constant.

In the three highest intensity segments, the inclusion of a constant significantly improved the fits (determined via an F-test for the inclusion of the extra component). Furthermore, in the segment with mean intensity 3458 counts/s, fitting without a constant resulted only in determining an upper limit on the peaked noise of $<3.1 \%$ (Table 4). This upper limit is rather large, and a visual inspection of the power spectrum showed peaked noise to be present. Therefore, it seems justified to include an extra component in the fits, without needing (nor being

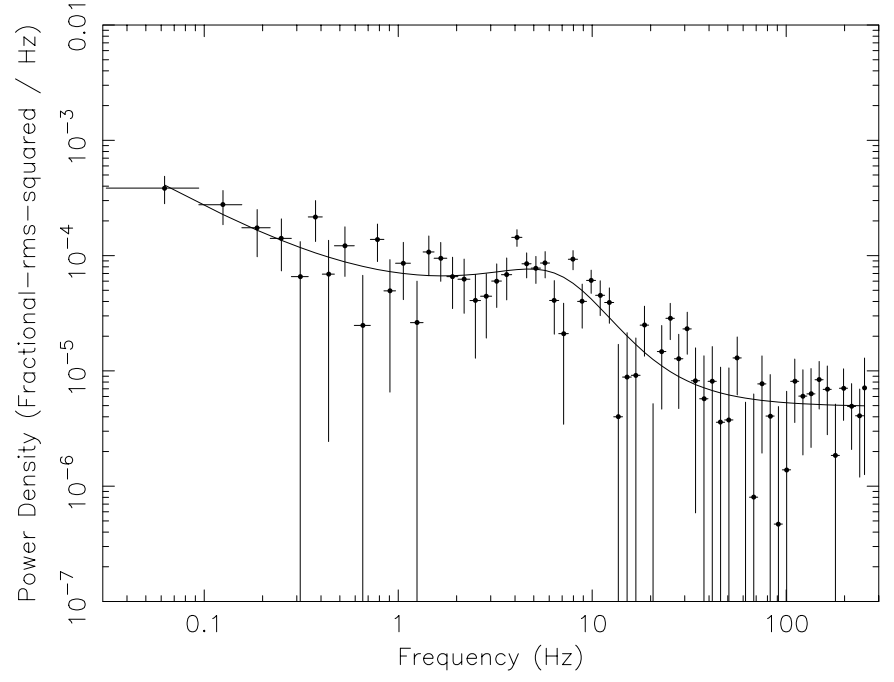

Fig. 7. PC power spectrum from the energy range $1.2-17.9 \mathrm{keV}$. The mean intensity is 3041 counts/s (mean rank number $\sim 1.06$; lower FB). The solid line shows the best fit, and includes a constant component

able) to describe the actual form of the possible higher frequency feature. We note that: HFN was reported in Sco X-2, with cut-off frequencies of $66 \mathrm{~Hz}$ and $41 \mathrm{~Hz}$ in the NB and FB respectively (HK); also a QPO with a frequency of $\sim 125 \mathrm{~Hz}$ and $F W H M$ of $\sim 30 \mathrm{~Hz}$ was detected by Penninx et al. (1990), in the FB of GX $17+2$.

The fact that we cannot determine the form of the higher frequency feature is not a major problem. The main result we obtained from the $\mathrm{PC}$ data is this: the peaked noise feature experienced a real weakening in the upper FB. This result is not sensitive to the presence or otherwise of a higher frequency feature.

\section{Discussion}

\subsection{Observed properties of Sco $X-2$}

We were able to trace the power spectral features of Sco $\mathrm{X}-2$ as a function of position in the $\mathrm{Z}$ track, in the energy range $\sim 1-17 \mathrm{keV}$. At no time did we detect a narrow $\mathrm{N} / \mathrm{FBO}$ as found in Sco X-1. Broad peaked noise was present at $\sim 4-7 \mathrm{~Hz}$, with $F W H M \sim 6-12 \mathrm{~Hz}$. We will subsequently refer to this feature as flaring branch noise (FBN). FBN was detected in the lower NB and reached a maximum at rank number $1.09(\sim 10 \%$ of the way up the $\mathrm{FB})$. At its maximum, the fractional rms was $\sim 3 \%$. It then became weaker and was undetectable by rank number 1.5 (the middle of the FB; upper limit 1.3\%). We found no significant change (more than a few $\mathrm{Hz}$ ) in the centroid frequency or $F W H M$ as the source moved up the FB. The FBN was stronger at higher energies.

The behaviour we have observed is very similar to that reported by Ponman et al. (1988). In addition, we have been able to unambiguously separate NB data from lower FB data. The increase in the fractional rms that accompanied movement from the NB through to the lower FB, 
Table 4. Results from fits to the PC power spectra from the energy range $1.2-17.9 \mathrm{keV}$

\begin{tabular}{|c|c|c|c|c|c|c|c|}
\hline \multirow{2}{*}{$\begin{array}{c}\text { Mean Intensity } \\
\text { (cnts/s/4-detectors) }\end{array}$} & \multicolumn{2}{|c|}{ VLFN } & \multicolumn{3}{|c|}{ Peaked Noise } & \multirow[t]{2}{*}{ Constant } & \multirow[t]{2}{*}{ Reduced $\chi^{2} /$ dof } \\
\hline & Index & rms (\%) & Frequency $(\mathrm{Hz})$ & $F W H M(\mathrm{~Hz})$ & $\mathrm{rms}(\%)$ & & \\
\hline \multicolumn{8}{|c|}{ Fits without an extra constant } \\
\hline $3041 \pm 6$ & $-0.5 \pm 0.1$ & $1.1 \pm 0.1$ & $5.4_{-0.7}^{+0.9}$ & $7.2_{-3.1}^{+2.7}$ & $2.5 \pm 0.4$ & - & $1.11 / 58$ \\
\hline $3458 \pm 14$ & $-0.5 \pm 0.1$ & $1.2 \pm 0.1$ & $5.4^{\mathrm{a}}$ & $7.2^{\mathrm{a}}$ & $<3.1$ & - & $1.26 / 61$ \\
\hline $4962 \pm 25$ & $-1.3 \pm 0.2$ & $2.0 \pm 0.3$ & $5.4^{\mathrm{a}}$ & $7.2^{\mathrm{a}}$ & $<1.8$ & - & $1.23 / 61$ \\
\hline $5492 \pm 31$ & $-1.4 \pm 0.2$ & $2.7_{-0.4}^{+0.5}$ & $5.4^{\mathrm{a}}$ & $7.2^{\mathrm{a}}$ & $<1.3$ & - & $1.44 / 61$ \\
\hline \multicolumn{8}{|l|}{ Fits with an extra constant } \\
\hline $3041 \pm 6$ & $-1.0_{-0.4}^{+0.2}$ & $1.0 \pm 0.1$ & $5.0_{-1.8}^{+0.8}$ & $10.3_{-2.5}^{+3.0}$ & $3.3_{-0.5}^{+0.4}$ & $4.8 \mathrm{e}-6_{-1.3 e-6}^{+1.0 \mathrm{e}-6}$ & $1.04 / 57$ \\
\hline $4962 \pm 25$ & $-1.5 \pm 0.2$ & $2.2 \pm 0.3$ & $5.9^{-1.1}$ & $11.9^{\mathrm{a}}$ & $<1.6$ & $3.6 \mathrm{e}-6 \pm 1.1 \mathrm{e}-6$ & $1.06 / 60$ \\
\hline $5492 \pm 31$ & $-1.6 \pm 0.2$ & $3.1^{+0.5}$ & $5.9^{\mathrm{a}}$ & $11.9^{\mathrm{a}}$ & $<1.2$ & $4.3 e-6 \pm 0.9 e-6$ & $1.10 / 60$ \\
\hline
\end{tabular}

${ }^{a}$ Fixed parameter.

was also seen in the RXTE data of KK. We have not been able to confirm the decrease that they observed in the centroid frequency and $F W H M$, which accompanied that transition.

\subsection{Comparison with other power spectral features}

The fast-time variability of Sco X-2 is different to that seen in the other $\mathrm{Z}$ sources. Most obvious is the low coherence of FBN when compared with N/FBOs. The FBN has a relative width of $\sim 1-2$, compared with the N/FBO in Sco X-1 which has a relative width of less than $\sim 0.5$ (Dieters \& van der Klis 2000). Also in Sco X-1, the frequency and $F W H M$ of the NBO increase with increasing inferred mass accretion rate, and there is a sudden jump in frequency at the NB/FB vertex. In Sco X-2 on the other hand, there is a decrease in the FBN centroid frequency and $F W H M$ with movement through the vertex (KK). In Sco X-1 the NBO strength increases with increasing rank number; the NBO becomes indistinguishable from the underlying noise (by $\sim 10 \%$ of the way up the FB) because the $F W H M$ increases. In comparison, FBN is strongest at about $10 \%$ of the way up the $\mathrm{FB}$, and becomes undetectable because of a subsequent decrease in the fractional rms.

Even given the marked differences, there are some similarities between FBN and the N/FBO seen in Sco X-1: the centroid frequencies are similar, both the N/FBO and FBN are hard, and there is no time lag between the soft and hard energies in either phenomenon. In Sco X-1, the $\mathrm{NB} / \mathrm{FB}$ vertex is a critical position in the $\mathrm{Z}$ track. In Sco X-2 there are hints that this may also be true, although in a different sense (i.e. frequency jump for NBOs compared with a frequency fall for FBN). FBN and $\mathrm{Z}$ source $\mathrm{N} / \mathrm{FBO}$ s may still be related, as already suggested by KK.

In the scheme of the unified model, radiation pressure drives matter up out of the disc, forming an inner disc corona, resulting in a radial inflow (Lamb 1991; Psaltis et al. 1995). When $\dot{M}$ rises to within $\sim 20 \%$ of the Eddington limit, radial oscillations in the optical depth of the inflowing material produce a rocking of the X-ray spectrum; this gives rise to NBOs (Fortner et al. 1989; Miller \& Park 1995). This model may explain the evolution of the N/FBO seen in Sco X-1, but it does not do well in explaining FBN. The FBN centroid frequency does not increase as predicted by the model. Furthermore, we have detected it $\sim 40 \%$ of the way up the FB; oscillations are predicted to be suppressed at such high accretion rates.

QPOs at super-Eddington accretion rates have been observed before: Kuulkers \& van der Klis (1995b) observed a $\sim 26 \mathrm{~Hz}$ QPO in the upper FB of Cyg X-2. The QPO was observed during an intensity dip in the $\mathrm{FB}$, and it was stronger at lower energies. They suggested that it is produced by oscillations in an inner disc torus, and it is observable because Cyg X-2 is viewed at a high inclination (see Sect. 5.3). Sco X-2, on the other hand, is thought to be viewed at a low inclination (see Sect. 5.3); therefore it is unlikely that FBN is related to the $26 \mathrm{~Hz}$ QPO.

KK pointed out that the FBN seen in Sco X-2 looks similar to peaked HFN sometimes seen in atoll sources. We observed a decrease in the FBN fractional rms as the source moved up the NB, which is opposite to what would be expected if the FBN was infact atoll source HFN. The low Nyquist frequency is no problem here as the break frequency of HFN decreases with decreasing inferred massaccretion rate (Wijnands \& van der Klis 1999). The phenomenology of FBN is thus significantly different to atoll source HFN; therefore we propose that FBN is unrelated to atoll source HFN.

\subsection{Two types of $Z$ sources: $A$ clue to the origin of FBN?}

In order to understand why Sco X-2 behaves so differently, it is worthwhile to investigate the general relationships that exist between the $\mathrm{Z}$ sources. On the basis of their Z track morphology and fast-time variability, they can be divided into two groups: Cyg X-2, GX 5-1, GX 340+0 (Cyg-like objects); and Sco X-1, Sco X-2, GX $17+2$ (Sco-like objects) (e.g. Kuulkers et al. 1997). The Cyg-like sources have much larger HBs and smaller FBs than the Sco-like sources. The HBs in the Sco-like objects are slanted, while the HBs in the Cyg-like sources 
are horizontal. Most importantly, in the Cyg-like objects the intensity is seen to fall in the middle and upper FB, while the Sco-like objects really do flare in the FB.

At high accretion rates an inner disc torus may be produced, which in high inclination sources can partly obscure the X-ray emitting region. The Cyg-like objects have thus been interpreted as being viewed at higher inclinations, and the Sco-like objects at lower inclinations (Kuulkers et al. 1997). In support of this, optical observations place Cyg X-2 at an inclination of $62.5 \pm 4^{\circ}$ (Orosz \& Kuulkers 1999), Sco X-1 at $15^{\circ}-40^{\circ}$ (Crampton et al. 1976), and also suggest that Sco X-2 is viewed at a low inclination (Wachter \& Margon 1996). Psaltis et al. (1995) have modelled the X-ray spectra of $\mathrm{Z}$ sources. They suggested that the Sco-like objects have weaker magnetic fields than the Cyg-like objects.

The most obvious difference between Sco X-2 and the other Z sources is the absence of an HB, so it is tempting to think that either: Sco X-2 has a weaker magnetic field than Sco X-1, while also (perhaps incidentally) being viewed at a low inclination; or simply that Sco X-2 never reaches a low enough mass-accretion rate to trace out an HB.

A close investigation of the timing properties of Cyg X-2 may provide hints as to the origin of FBN. In Cyg X-2 long-term variations in the mean X-ray intensity have been discovered (e.g. Paul et al. 2000). Ginga observations of $\mathrm{Cyg} \mathrm{X}-2$ showed that the $\mathrm{Z}$ track was more Sco-like at medium level intensities, and more Cyg-like at high level intensities (Wijnands et al. 1997). RXTE observations of Cyg X-2 at low intensities revealed no NBO (upper limit $\sim 1 \%$ ) in the NB; however a peaked noise feature was detected in the lower $\mathrm{FB}$, with a frequency and fractional $\mathrm{rms}$ of $6-7 \mathrm{~Hz}$ and $\sim 3 \%$ respectively (Kuulkers et al. 1999). This is (perhaps only superficially) similar to the FBN seen in Sco X-2.

It is vital to have more observations of Cyg X-2 at low intensities so that we can better understand the properties of the peaked noise feature. Only then can we make any conclusions about the relationship between FBN and the Cyg X-2 peaked noise. But if the two features are the same phenomenon, we must ask: what are the physical characteristics of Cyg X-2 when it is at low level intensities? The low level may be due to obscuration by the outer accretion disc, possibly caused by a precessing disc (e.g. Kuulkers et al. 1996). Wijnands \& van der Klis (2001) have argued against the precessing disc model on the basis of differing $\mathrm{kHz}$ QPO properties at different intensity levels. Kuulkers et al. (1999) showed that the relative VLFN properties at different intensity levels were also inconsistent with obscuration. Instead, they preferred a model in which the overall intensity level is sensitive to the properties of the inner mass flow. Whatever the case, if FBN and Cyg X-2 peaked noise are produced by the same process, then that process will be largely insensitive to orbital inclination. In this way, Sco X-2 may prove to be a useful tool in discerning between models trying to explain the behaviour of Cyg X-2, and ultimately the other Z sources.

\section{Conclusions}

We have carried out the first detailed study of the X-ray fast-time variability of the Z source Sco X-2, as a function of position in the $\mathrm{Z}$ track. We found broad peaked noise with centroid frequency and $F W H M$ in the range $\sim 4-7 \mathrm{~Hz}$ and $\sim 6-12 \mathrm{~Hz}$ respectively. We find it difficult to attribute FBN to the same processes which are able to explain typical Z source N/FBOs. We also conclude that it is not a manifestation of atoll source HFN. FBN resembles the peaked noise seen in the FB of Cyg X-2 at low overall intensities. If these are later confirmed to be the same phenomena, then the process responsible for FBN must be largely insensitive to orbital inclination.

Acknowledgements. This research has made use of data obtained from the Leicester Database and Archive Service at the Department of Physics and Astronomy, Leicester University, UK. The reduction of raw data was carried out with support from the Cooperative Research Centre for Advanced Computational Systems established under the Australian Government's Cooperative Research Centres Program. PMO wishes to thank the Space Research Organization Netherlands for its hospitality, and is currently being supported by the Australian Postgraduate Award scheme.

\section{Appendix: Calculation of deadtime-affected white-noise levels}

In the absence of deadtime, the white-noise level in a Leahy normalised power spectrum, due to Poisson noise in the data, is equal to 2 (Leahy et al. 1983). The effect of deadtime is to decrease the variance of the data and thus lower the white-noise level. The deadtime-affected whitenoise level for the sum channel $P_{\nu \mathrm{s}}$, can be calculated for each frequency $\nu$ in the power spectrum

$$
\begin{aligned}
& <P_{\nu \mathrm{s}}>=2\left(1-\mu_{\mathrm{s}} \tau_{\text {dead }}\right)^{2} \times \\
& \left(1+2\left(\frac{\mu_{\mathrm{s}} \tau_{\text {dead }}}{1-\mu_{\mathrm{s}} \tau_{\text {dead }}}\right)\left(\frac{\tau_{\text {dead }}}{\tau_{\text {res }}}\right) \sin ^{2}\left(\frac{\pi \nu}{2 \nu_{\text {Nyquist }}}\right)\right)
\end{aligned}
$$

where $\mu_{\mathrm{s}}$ is the observed counting rate per detector, $\tau_{\text {res }}$ is the time resolution of the lightcurve, $\tau_{\text {dead }}$ is the deadtime per event, and $\nu_{\text {Nyquist }}$ is the Nyquist frequency of each transform (Weisskopf 1985).

The deadtime-affected white-noise level for any particular energy band $P_{\nu \mathrm{b}}$, can be calculated as a function of $P_{\nu \mathrm{s}}$. For an arbitrary time interval, the ratio of the total number of counts $k$ in the particular energy band, to the total number of counts $n$ in the sum channel is denoted by $r$. The mean variance of the data is denoted as $\sigma_{\mathrm{s}}^{2}$ and $\sigma_{\mathrm{b}}^{2}$ for the sum channel and the particular energy band respectively. These are related by (Mitsuda \& Dotani 1989)

$\sigma_{\mathrm{b}}^{2}=r^{2} \sigma_{\mathrm{s}}^{2}+k(1-r)$.

Each transform is calculated from a time series $x_{k}(k=$ $0, \ldots, N-1)$ consisting of $N$ arbitrary time intervals. By employing Parseval's theorem, we can relate the total 
variance $\operatorname{Var}\left(x_{k}\right)$ (or the mean variance $\sigma^{2}$ ) of the time series, and the Fourier amplitude $a$

$\operatorname{Var}\left(x_{k}\right)=N \sigma^{2}=\frac{N-1}{N}|a|^{2}$.

Here, we have considered only situations where the dependence of Fourier amplitude on frequency is negligible (i.e. white-noise). The factor of $N-1$ originates from the fact that the sum used in the expression for the total variance does not include a term for a frequency of zero.

The mean variance $\sigma^{2}$ and Leahy power $P$ can then be related via

$\sigma^{2}=P \frac{N_{\mathrm{ph}}}{2}\left(\frac{N-1}{N^{2}}\right)$.

$N_{\text {ph }}$ is equal to $N n$ for the sum channel or $N k$ for the particular energy band. We can express $P_{\mathrm{b}}$ as a function of $P_{\mathrm{s}}$ by substituting Eq. (A.4) into Eq. (A.2)

$P_{\mathrm{b}} \frac{k}{2}\left(\frac{N-1}{N}\right)=P_{\mathrm{s}} \frac{k^{2}}{n^{2}} \frac{n}{2}\left(\frac{N-1}{N}\right)+k(1-r)$

or

$P_{\mathrm{b}}=2\left(\frac{N}{N-1}\right)-\frac{k}{n}\left(2\left(\frac{N}{N-1}\right)-P_{\mathrm{s}}\right)$

which in the case of large $N$ reduces to

$P_{\mathrm{b}}=2-\frac{k}{n}\left(2-P_{\mathrm{s}}\right)$.

\section{References}

Cowley, A. P., \& Crampton, D. 1975, ApJ, 201, L65

Crampton, D., Cowley, A. P., \& Hutchings, J. B., et al. 1976, ApJ, 207, 907

Dieters, S. W., \& van der Klis, M. 2000, MNRAS, 311, 201

Dieters, S. W., Vaughan, B. A., Kuulkers, E., et al. 2000, A\&A, 353, 203

Fortner, B., Lamb, F. K., \& Miller, G. S. 1989, Nature, 342, 775

Hasinger, G., \& van der Klis, M. 1989, A\&A, 225, 79

Hasinger, G., van der Klis, M., \& Ebisawa, K. 1990, A\&A, 235, 131

Hertz, P., Vaughan, B., Wood, K. S., et al. 1992, ApJ, 396, 201

Kuulkers, E., \& van der Klis, M. 1995a, Ann. New York Ac. Sci., 759, 344

Kuulkers, E., \& van der Klis, M. 1995b, A\&A, 303, 801
Kuulkers, E., \& van der Klis, M. 1998, A\&A, 332, 845

Kuulkers, E., van der Klis, M., Oosterbroek, T., et al. 1994, A\&A, 289, 795

Kuulkers, E., van der Klis, M., \& Vaughan, B. A. 1996, A\&A, 311,197

Kuulkers, E., van der Klis, M., Oosterbroek, T., et al. 1997, MNRAS, 287, 495

Kuulkers, E., Wijnands, R., \& van der Klis, M. 1999, MNRAS, 308,485

Lamb, F. K. 1991, in Unified Model of X-ray Spectra and QPOs in Low Mass Neutron Star Binaries, Neutron Stars: Theory and Observation, ed. J. Ventura, \& D. Pines (Dordrecht, Kluwer Academic Publishers), 445

Leahy, D. A., Darbo, W., Elsner, R. F., et al. 1983, ApJ, 266, 160

Lewin, W., Lubin, L., Tan, J., et al. 1992, MNRAS, 256, 545

Makino, F., \& ASTRO-C Team 1987, Astrophys. Lett. Communic., 25, 223

Miller, G. S., \& Park, M.-G. 1995, ApJ, 440, 771

Mitsuda, K., \& Dotani, T. 1989, PASJ, 41, 557

Orosz, J. A., \& Kuulkers, E. 1999, MNRAS, 305, 132

Paul, B., Kitamoto, S., \& Makino, F. 2000, ApJ, 528, 410

Penninx, W., Lewin, W. H. G., \& Mitsuda, K., et al. 1990, MNRAS, 243, 114

Ponman, T. J., Cooke, B. A., \& Stella, L. 1988, MNRAS, 231, 999

Psaltis, D., Lamb, L., \& Miller, G. 1995, ApJ, 454, L137

Schultz, N. S., Hasinger, G., \& Trümper, J. 1989, A\&A, 225, 48

Turner, M., Thomas, H., Patchett, B., et al. 1989, PASJ, 41, 345

van der Klis, M. 1989, in Fourier Techniques in X-ray Timing, Timing Neutron Stars, ed. H. Ögelman, \& E. van den Heuvel (Kluwer Academic Publishers, Dordrecht), 27

van der Klis 1995, in Rapid Aperiodic Variability in X-ray Binaries, ed. W. H. G. Lewin, J. van Paradijs, \& E. P. J. van den Heuvel (Cambridge University Press, Cambridge), 252

van der Klis 2000, ARA\&A, 38, 717 [astro-ph/0001167]

Wachter, S. 1997, BAAS, 191, 4411

Wachter, S., \& Margon, B. 1996, AJ, 112, 2684

Weisskopf, M. C. 1985, talk presented at workshop on Time Variability in X-ray and Gamma-Ray Sources, Taos, New Mexico

Wijnands, R., \& van der Klis, M. 1999, ApJ, 514, 939

Wijnands, R., \& van der Klis, M. 2001, MNRAS, 321, 537

Wijnands, R. A. D., van der Klis, M., \& Kuulkers, E., et al. 1997, A\&A, 323, 399 